\title{
DIOS: the dark baryon exploring mission
}

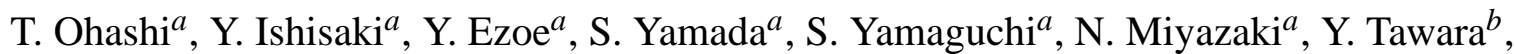

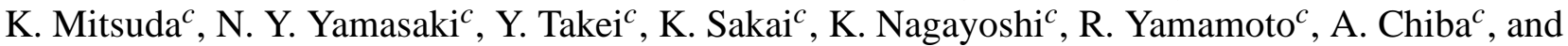 \\ T. Hayashi ${ }^{c}$ \\ ${ }^{a}$ Department of Physics, Tokyo Metropolitan University, 1-1 Minami-Osawa, Hachioji, \\ Tokyo 192-0397, Japan \\ ${ }^{b}$ Depertment of Phycics, Nagoya University, Furo-cho, Chikusa, Nagoya 464-8602, Japan \\ ${ }^{c}$ Institute of Space and Astronautical Science, Japan Aerospace Exploration Agency, 3-1-1, \\ Yoshinodai, Chuo, Sagamihara, Kanagawa 252-5210, Japan
}

\begin{abstract}
DIOS (Diffuse Intergalactic Oxygen Surveyor) is a small satellite aiming for a launch around 2020 with JAXA's Epsilon rocket. Its main aim is a search for warm-hot intergalactic medium with high-resolution X-ray spectroscopy of redshifted emission lines from OVII and OVIII ions. The superior energy resolution of TES microcalorimeters combined with a very wide field of view (30-50 arcmin diameter) will enable us to look into gas dynamics of cosmic plasmas in a wide range of spatial scales from Earth's magnetosphere to unvirialized regions of clusters of galaxies. Mechanical and thermal design of the spacecraft and development of the TES calorimeter system are described. We also consider revising the payload design to optimize the scientific capability allowed by the boundary conditions of the small mission.
\end{abstract}

Keywords: intergalactic medium, X-ray spectra, oxygen lines, microcalorimeters, mechanical coolers, X-ray telescope

\section{INTRODUCTION}

Majority of baryons in the local universe remain unexplored and are called as dark baryons. Baryon census has been carried out based on the observed contents of widespread medium around galaxies, clusters, and in large-scale structures. However, $30-50 \%$ of the baryons are still missing. ${ }^{1}$ Numerical simulations and recent observations indicate that dark baryons are likely to reside along the large-scale filaments in the form of warm-hot intergalactic medium (WHIM) with temperatures between $10^{5}$ and $10^{7} \mathrm{~K}$. The density of WHIM is 10-100 times that of the average level in the universe, and even lower than those in the virial radii of clusters of galaxies by a factor of up to 10. This makes the direct detection of WHIM very difficult. Note that UV observations of OVI and other absorption lines from FUSE and HST (COS in particular) have given firm evidences that there indeed exists a warm gas with temperatures $\sim 10^{5} \mathrm{~K}$ in the intergalactic space. ${ }^{1}$

Since main part of WHIM is in a temperature range of a few times $10^{6} \mathrm{~K}$, the most efficient observation will be in X-rays, either by emission or absorption lines. There are a few cases where absorption by the WHIM is observed significantly with grating spectrometers on Chandra and XMM-Newton. ${ }^{2,3}$ As for the WHIM detection in emission, the low surface brightness makes the study particularly difficult. However, X-ray emission from WHIM will tell us about its spatial distribution at different redshifts and will show us the evolution of the large-scale structure of the universe in a direct way.

The concept and design of DIOS (Diffuse Intergalactic Oxygen Surveyor) has been reported in the past SPIE conference papers for several times. ${ }^{4-7}$ A special feature of the DIOS mission is a wide-field X-ray spectroscopy using an array of microcalorimeters, enabling the detection of WHIM in emission. A detailed simulation has been carried out assuming a few times larger X-ray telescope, ${ }^{8}$ and the results can be easily scaled to DIOS observations since influence of non-X-ray background will be negligible. Technology of X-ray microcalorimeters has been well established toward the launch of ASTRO-H in late 2015. ${ }^{9}$ This mission will be the first satellite to carry out cosmic X-ray observations with microcalorimeters. New technologies which enable the space application of microcalorimeters have been developed: such as Joule-Thomson coolers backing up liquid He cooling, 3-stage ADR (adiabatic demagnetization refrigerator) maintaining

Further author information: (Send correspondence to T. Ohashi.) T. Ohashi: E-mail: ohashi@tmu.ac.jp, Telephone: +81-426-77-2492 
the detector temperature at $50 \mathrm{mK}$, an on-board pulse shape analysis system, and X-ray generators enabling continuous calibration in the orbit.

The unique capability of microcalorimeters is the high-resolution spectroscopy of extended sources, in contrast to grating spectrometers. ASTRO-H SXS experiment gives a field of view of $3 \times 3 \mathrm{arcmin}$, and studies of much more extended objects will have to await further development of, such as, a TES calorimeter array. This type of instrument is a baseline instrument for Athena, the large X-ray observatory planned for launch in 2028 by ESA. DIOS will provide a large field of view (about 50 arcmin) by combining TES calorimeters with a short focal length telescope. X-ray spectroscopy of extended objects from earth's environment to cluster outskirts will be carried out in a very efficient way. Thermalization processes, shock formation, turbulence, resonance scattering and charge exchange process will all be examined in a clear way.

In this paper, we give an updated status of the DIOS mission over the previous reports. We plan to propose DIOS to the 4th mission in the JAXA series of small project using Epsilon rocket for the launch. JAXA's framework for small missions has been revised in 2013 with the release of a new roadmap of space science and space exploration in Japan. We will discuss possible ways of enhancing the original capability of DIOS within the available payload specifications. We note that DIOS is along the line of continuous effort of an international collaboration which so far has proposed dark-baryon missions to ESA's Cosmic Vision (EDGE and ORIGIN) and to the US Decadal Survey (Xenia). ${ }^{10-13}$

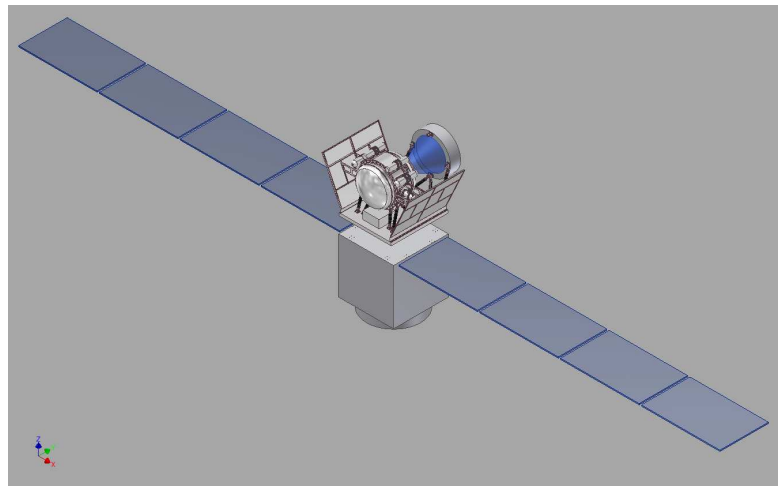

Figure 1. The DIOS spacecraft. The length of the solar paddle is $10 \mathrm{~m}$ after it is deployed. Total mass will be about $615 \mathrm{~kg}$.

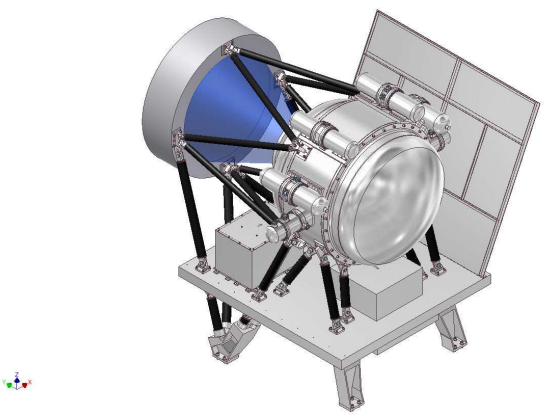

Figure 2. Close-up view of the payload of DIOS, showing the $\mathrm{X}$-ray telescope and dewar. The focal length of the telescope is $70 \mathrm{~cm}$.
Table 1. Parameters of the DIOS spacecraft

\begin{tabular}{|l|l|}
\hline Total mass & $615 \mathrm{~kg}$ \\
Payload mass & $323 \mathrm{~kg}$ \\
Size at launch & $1.2 \times 1.45 \times 1.4 \mathrm{~m}$ \\
Size in orbit & $5.9 \times 1.45 \times 1.4 \mathrm{~m}$ \\
Attitude control & 3 -axis \\
Pointing accuracy & $\leq 30$ arcsec \\
Total power & $691 \mathrm{~W}$ \\
Payload power & $381 \mathrm{~W}$ \\
\hline
\end{tabular}

(1)

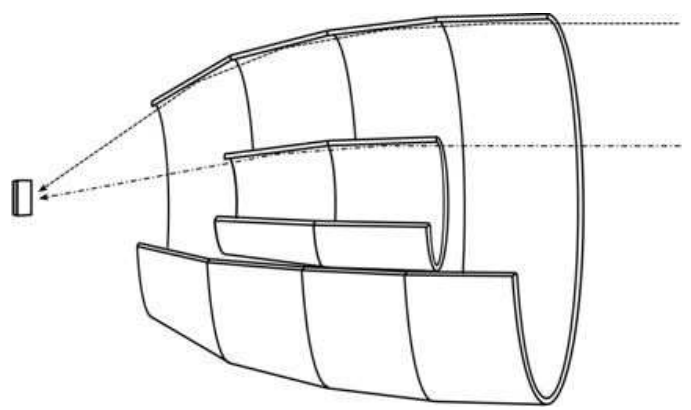

Figure 3. Concept of the four-reflection X-ray telescope for DIOS.

\section{SPACECRAFT}

The view of the DIOS spacecraft is shown in Fig. 1, and main parameters of the satellite are summarized in Table 1. The spacecraft will weigh about $600 \mathrm{~kg}$, with the payload mass $\sim 320 \mathrm{~kg}$. The total spacecraft power will be $\sim 700 \mathrm{~W}$, of which about $380 \mathrm{~W}$ will be used by the payload. The cryocoolers (2-stage Stirling coolers and Joule-Thomson coolers) 
will require power around $300 \mathrm{~W}$. The orbit of DIOS will be a low-earth circular one with an altitude of about $550 \mathrm{~km}$, same as those of all the previous Japanese X-ray satellites including Suzaku. The launch will take place at USC (Uchinoura Space Center, $131.1^{\circ} \mathrm{E}, 31.1^{\circ} \mathrm{N}$ ) in Kagoshima prefecture, Japan, giving the orbital inclination to be $31^{\circ}$.

The spacecraft attitude will be 3-axis stabilized. Since angular resolution of the X-ray instrument will be about 3', DIOS will not need very fine pointing accuracy. To reconstruct the attitude accurately enough for the data analysis, star trackers will be equipped and give the attitude information with about $10^{\prime \prime}$ accuracy. The baseline design has the telescope direction perpendicular to the sun direction, and accessible sky region will be along a great circle with $90^{\circ} \pm 25^{\circ}$ from the sun direction. Radiator panels with an approximate area of $1 \mathrm{~m}^{2}$ will exhaust heat from the spacecraft. Thermal analysis indicates that heat input when the radiator points to the earth will not be a serious problem, so switching of two radiator panels is not an absolute necessity.

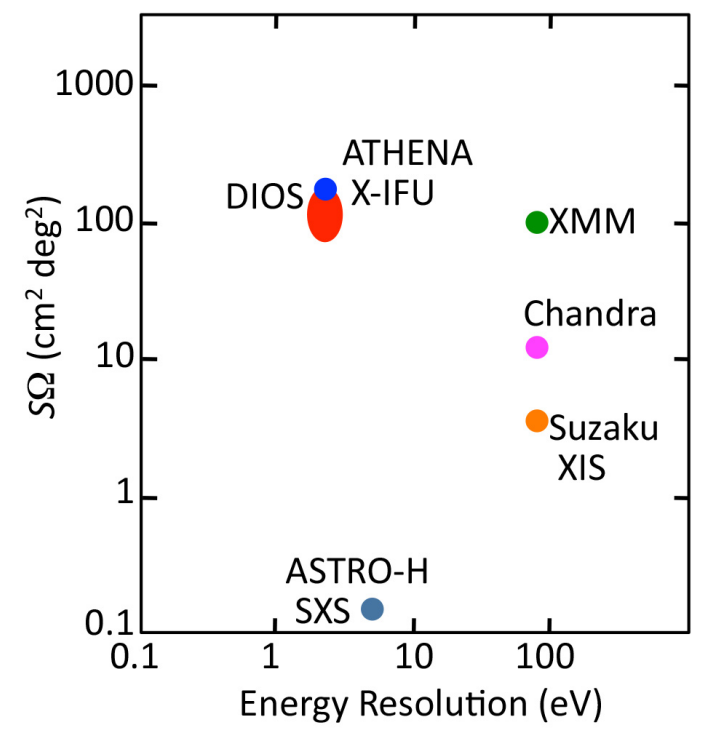

Table 2. Parameters of the observing instruments on board DIOS

\begin{tabular}{|l|l|}
\hline Effective area & $200 \mathrm{~cm}^{2}$ \\
Field of view & $50^{\prime}$ diameter \\
$S \Omega$ & $\sim 150 \mathrm{~cm}^{2} \mathrm{deg}^{2}$ \\
Angular resolution & $3^{\prime}(16 \times 16$ pixels $)$ \\
Energy resolution & $<5 \mathrm{eV}(\mathrm{FWHM})$ \\
Energy range & $0.1-1.5 \mathrm{keV}$ \\
Observing life & $>5 \mathrm{yr}$ \\
\hline
\end{tabular}

Figure 4. Comparison of $S \Omega$ and energy resolution for spectroscopic instruments (CCDs and microcalorimeters) for planned and operating X-ray satellites.

A close-up view of the payload part is shown in Fig. 2. The payload consists of a four reflection telescope (FXT whose design concept is shown in Fig. 3) and a dewar containing X-ray spectrometer array (XSA) consisting of TES microcalorimeters. Focal length of FXT is $70 \mathrm{~cm}$ in the baseline design. FXT and dewar are connected and supported from the baseplate by CFRP torus structures. The baseplate also provides an interface between the payload part and the satellite bus. The baseplate will be equipped with electronics boxes and a radiator panel will stand out with an angle not directly looking into solar paddles. The dewar is similar to but smaller than the ASTRO-H one since no liquid He will be used in DIOS. Thermal and mechanical analysis has been carried out for a simplified model. We confirmed that cooler powers already confirmed for ASTRO-H will be high enough to cool the DIOS instrument with an input power of $280 \mathrm{~W}$. Also, the characteristic frequency of the payload is within the acceptable range. We plan to launch the spacecraft in warm condition, and to spend a few weeks to cool down the payload in space. In order to generate the total power of about 700 $\mathrm{W}$, the solar paddle consists of 4 panels on both sides.

Fig. 4 shows $S \Omega$ or grasp for various missions against energy resolution, and Table 2 shows parameters of the DIOS instrument. Energy resolution needs to be better than $5 \mathrm{eV}$ to resolve red-shifted WHIM emission lines from those of Galactic or solar-system emission. DIOS will provide a factor of $\sim 250$ improvement over ASTRO-H SXS in $S \Omega$, which is close to the level of Athena X-IFU. With such a small satellite, DIOS will offer a very high sensitivity to extended X-ray emission. 


\section{INSTRUMENT DEVELOPMENT}

\subsection{X-ray telescope}

Development of FXT (4-reflection X-ray telescope) in Nagoya University was started early and has been reported for a number of times ${ }^{14-16}$ as well as in the present conference (9144-235). A schematic view of FXT is shown in Fig. 3. We summarize its performance briefly. The focal length is $70 \mathrm{~cm}$, enabling a small focal-plane instrument to cover a large sky area and leading to very low detector background. The solid angle of the field of view of DIOS $\left(50^{\prime}\right)$ will be about 300 times larger than the SXS instrument on ASTRO-H. The goal of the angular resolution is $3^{\prime}$, noting that four reflections inevitably introduce additional image blurring than the 2 reflection mirror. An X-ray beam measurement of a test mirror set in a quadrant housing gave a half-power diameter of about 10 arcmin, and use of thicker foils $(0.2 \mathrm{~mm}$ rather than 0.15 $\mathrm{mm}$ ) is under consideration.

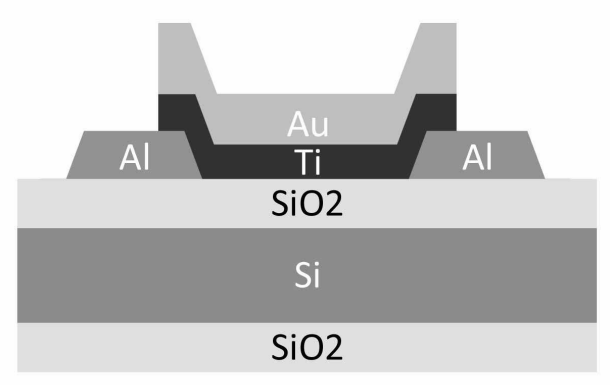

Figure 5. Cross sectional view of a TES pixel for the new layered wiring developed for TES array.

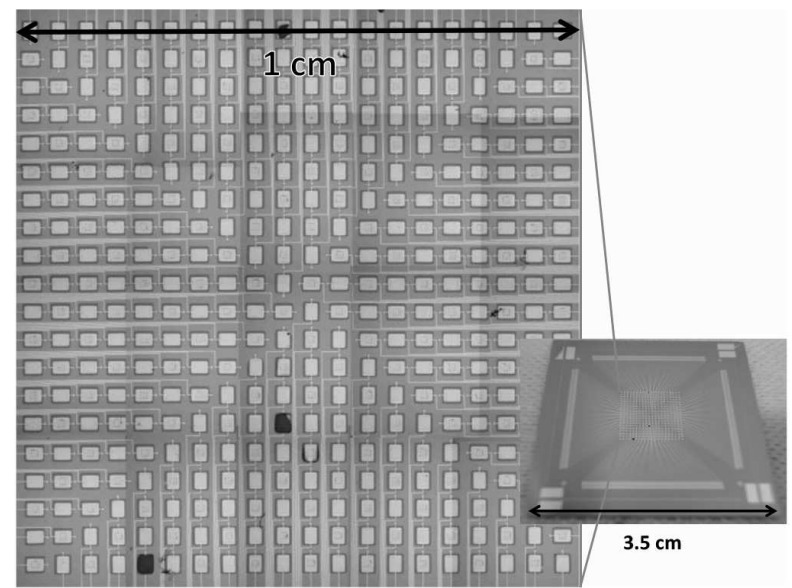

Figure 6 . A test model of TES calorimeter array with $20 \times 20$ pixels.

\subsection{TES array}

The focal plane instrument XSA (X-ray Spectrometer Array) is an array of TES microcalorimeters, whose development in Japan is a collaborative effort of Tokyo Metropolitan University, ISAS/JAXA, and Kanazawa University. Below, we briefly describe the status of our instrument development.

Since the wirings all running in the same plane will hamper dense layout of the TES calorimeter pixels, we have been developing multilayer wiring technique. ${ }^{17}$ A cross section of a TES pixel is shown in Fig. 5, and a test array with 400 pixels employing the multi-layer wiring is shown in Fig. 6. The hot and return lines run in different layers separated by an insulation layer. These wires make contact at a contact hole near each calorimeter pixel. The Si substrate has a thickness of $300 \mu \mathrm{m}$. The wirings are made of $\mathrm{Al}$, and their thicknesses are $50-100 \mathrm{~nm}$, and the insulation $\mathrm{SiO}_{2}$ layer has a thickness of $500 \mathrm{~nm}$. Width of the wirings is only 10 and $15 \mu \mathrm{m}$, and a very fine alignment is performed during the production. Our production process requires that the multi-layer wirings are built first in the Si substrate, and then we form TES calorimeters on top of these wirings.

Early practice showed some problems which required modification in the process of the array production. ${ }^{18}$ The wiring makes a step of about $50 \mathrm{~nm}$ height at the edge of the square pixel area, and simple deposition of Ti-Au bilayer causes damage of the bilayer structure due to a mechanical break at these steps. Because of this, we could not obtain a good superconducting transition performance, such as a dull $R-T$ curve.

To improve this, we introduced a slanted machining of the wall of the multi-layer wiring and created a slope of about $45^{\circ}$ as shown in Fig. 5. This additional machining is provided by National Institute of Advanced Industrial Science and Technology (AIST) in Japan. We find that forming of Ti-Au bilayer on this slope does not cause a mechanical break, and the quality of the bilayer is improved. A test sample with $40 \mathrm{~nm}$ thick Ti and $130 \mathrm{~nm}$ Au bilayer showed a significantly better transition properties with a transition temperature $175 \mathrm{mK}$, residual resistivity of $0.3 \mathrm{~m} \Omega$, and normal resistivity of 
$378 \mathrm{~m} \Omega$. Therefore, basic production method of TES array with multilayer wiring has been almost established. Next step is a production of actual TES array and its test with X-rays.

We performed an experiment to examine radiation tolerance of TES calorimeters. ${ }^{19}$ TES calorimeter with a Ti/Au bilayer of $30 / 40 \mathrm{~nm}$ thick attached with a $1.5 \mu \mathrm{m}$ thick Au absorber was irradiated by $150 \mathrm{MeV}$ proton beam with a total dose of $10 \mathrm{krad}$. This is approximately 10 years of irradiation in the low Earth orbit. We see no significant change in the transition temperature or the energy resolution, which is $5.6 \pm 0.3 \mathrm{eV}$ (FWHM) at $6 \mathrm{keV}$ after the irradiation and degraded by about $10 \%$. This indicates that our TES calorimeters have sufficient radiation tolerance in orbit.

\subsection{TES array readout}

Frequency Domain Multiplexing (FDM) method to handle the signal readout from TES arrays has been developed. ${ }^{7}$ TES pixels are AC-biased with different frequencies at an order of $\mathrm{MHz}$, and summed by a SQUID and de-multiplexed by room temperature electronics. We designed, fabricated and tested several key components.

Phase delay between the SQUID and the room temperature demultiplexing electronics limits the bandwidth of the SQUID to be less than $<1 \mathrm{MHz}$, and it affects the number of multiplexing channels with a standard flux-locked loop feedback. We adopt a base-band feedback (BBFB) method $^{20}$ to compensate the delay by adjusting the phase in the feedback loop. An analog electronics using phase sensitive detectors are developed and demonstrated for a 4-channel TES device at bias frequencies of $1-3 \mathrm{MHz}$. We successfully detected X-ray pulses simultaneously from the TES array, but deficiency of the loop-gain caused non-linearity in SQUID and produced crosstalks between TES pixels. ${ }^{21}$

Based on these experiments, we designed and manufactured a digital BBFB circuit using FPGA in order to demultiplex and trigger events ${ }^{22}$ as shown in Fig. 7. The loop-gain was improved to handle driving frequencies up to $7 \mathrm{MHz}$. The number of multiplex channels is limited by the bandwidth and resolution of DAC in the output feedback current. We tested the performance against simulated TES pulses for 16 channels with a 14-bit 250 Msps (Mega-samples per sec) ADC and a 16-bit $800 \mathrm{Msps} \mathrm{DAC}$, and obtained enough signal-to-noise ratio of $72 \mathrm{~dB}$ where $60 \mathrm{~dB}$ was required to achieve a $2 \mathrm{eV}$ resolution for pulses corresponding to $1 \mathrm{keV}$. The expected resolution is also shown in Fig. 7. For DIOS detector system, a 15 bit DAC is required to multiplex 16 channels by keeping sufficient energy resolution.

Suppression of power dissipation by SQUID is important, because it limits configuration of cold stage components and distance between SQUID and TES array. We developed SQUID series for this application whose power dissipation is as low as $\sim 30 \mathrm{nW}$ per channel with enough gain. ${ }^{22}$ Gradiometer at SQUID input-coil works effectively under the Earth's magnetism. LC-filter array is also fabricated on $2.5 \times 2.5 \mathrm{~mm}^{2}$ SQUID chips as shown in Fig. 8. 4 capacitors with different area are made with anodized Aluminum. In combination with $500 \mathrm{nH}$ coils, resonances between 5 and $6 \mathrm{MHz}$ are measured by a SQUID at the He temperature. End-to-end test which includes a TES array, on-chip resonator, low-power SQUID, and digital BBFB circuit are planned.

\section{THERMAL AND MECHANICAL DESIGN}

The cooling system of DIOS is the same as that employed for ASTRO-H, ${ }^{23}$ except that no liquid He will be used for DIOS. The cooling chain is shown in Fig. 9. We will keep the radiator panel with an area of $1.5 \mathrm{~m}^{2}$ at less than $210 \mathrm{~K}$. The first stage of the cooler is a 2-stage Stirling cooler which takes the temperature down to $15-20 \mathrm{~K}$. Then, ${ }^{4} \mathrm{He}$ Joule-Thomson cooler takes the temperature down to $4.5 \mathrm{~K}$. Cool-down tests have been performed for both engineering (EM) and flight (FM) models of the ASTRO-H dewar, and coolings without employing liquid He show good performances. No consumable cryogen will be used in the DIOS cooling system, and we expect to have a long observing life in the orbit if mechanical coolers continue to work. The DIOS coolers will incorporate all the experimental knowledge obtain in the test of ASTRO$\mathrm{H}$ coolers. In particular, micro-vibration influence to the TES detectors has been addressed and further reduction of this effect will be the subject of cooler development for DIOS. The XSA system will go through a warm launch, and we have to allow for the initial cooling of the system during the first 1 month or so in the orbit.

Based on the specifications of the ASTRO-H cooling system, the required power for the mechanical coolers is about $230 \mathrm{~W}$, including the nominal margin of $30 \%$. The total payload power, including ADR control and signal processing system, is about $380 \mathrm{~W}$. To cope with this power generation, baseline design of solar paddles consists of four panels on each side as shown in Fig. spacecraft. The mass of the payload is about $323 \mathrm{~kg}$, and the total spacecraft mass will be 615 $\mathrm{kg}$ which is within the capability of the current model of the Epsilon rocket. Since we can expect some increase in the

launch capability of the rocket by the 4th mission, which is the one we hope to launch DIOS in 2020, we may be able to consider a small upgrading regarding the size and spacecraft mass. 

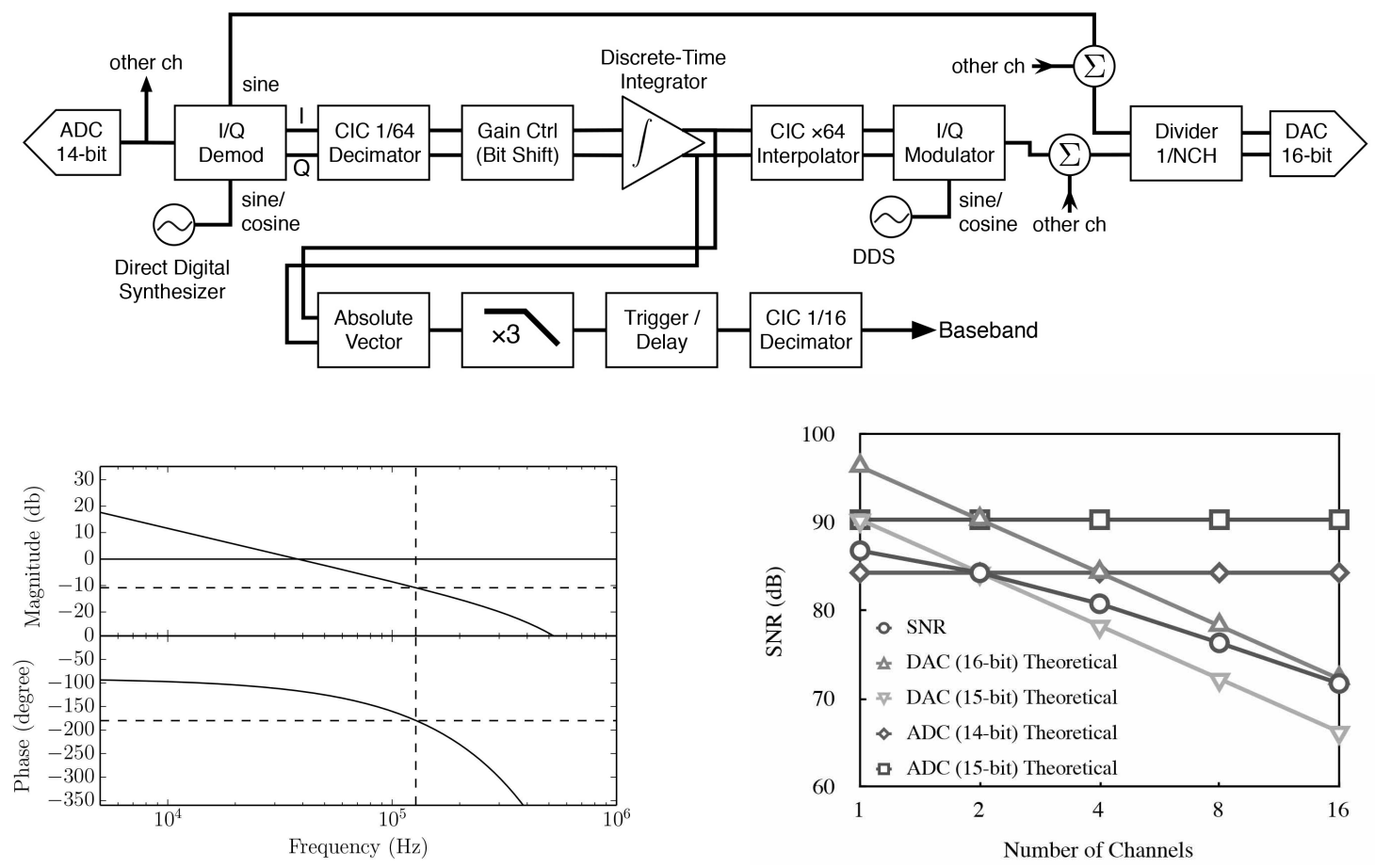

Figure 7. (Top) Current design of BBFB circuit using FPGA, (Bottom-left) Loop-gain and phase of current design. (Bottom-right) Signal-to-Noise ratio as a function of number of multiplexed channels with DAC/ADC resolution.
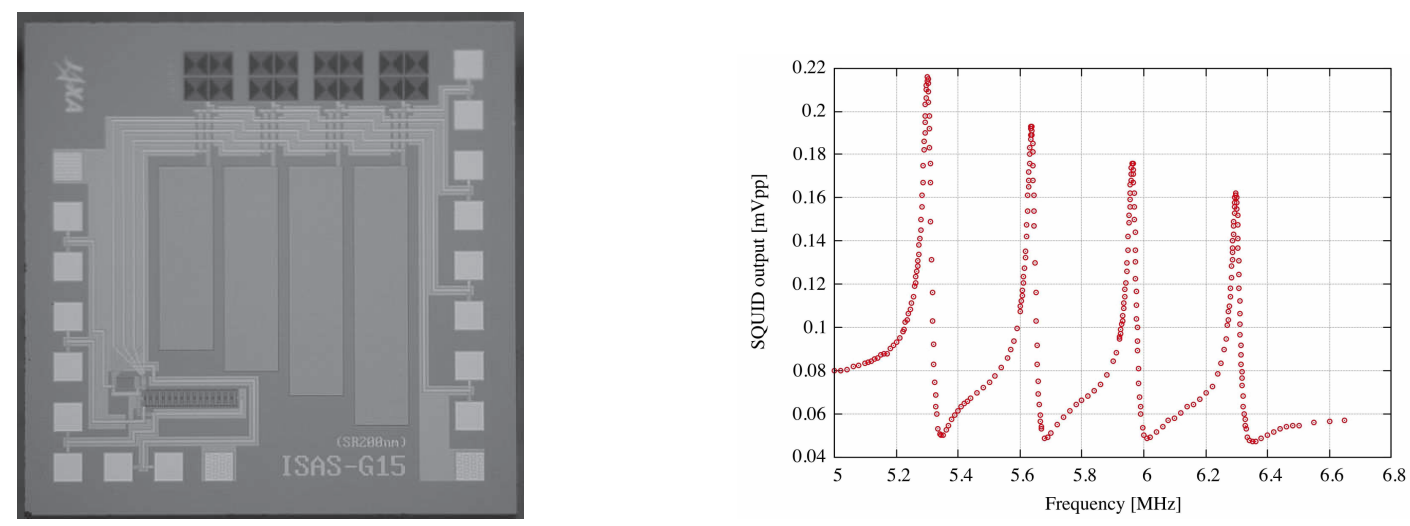

Figure 8. (Left) A picture of LC resonators on a $2.5 \times 2.5 \mathrm{~mm}$ chip. 4 capacitors of different area and $4500 \mathrm{nH}$ coils are fabricated with a SQUID array. (Right) Measured response of LC resonators at He temperature.

\section{SCIENCE FROM DIOS}

For WHIM observations, realistic simulations have been carried out by Takei et al. ${ }^{8}$ assuming instrument parameters for Xenia which has about 4 times larger collecting area than DIOS. Since contribution of non-X-ray background is expected be negligible, we may scale the results by Takei et al. by increasing the observing time. The simulation included the foreground emission and required simultaneous detection of both OVII and OVIII emission lines above $5 \sigma$. Detection of the two lines will allow us to estimate the average temperature of the emitting clouds. Even though the clouds are likely to be inhomogeneous with a range of temperature, our simulation shows that the derived temperature by assuming a thermal equilibrium is within $30 \%$ of the actual average temperature of the emitting region wighted by the OVII line intensity.

We will use energy ranges which are relatively free from foreground emission lines, and look for WHIM oxygen lines in the corresponding redshift intervals. The detected WHIM clouds from our simulation show many spatial concentrations 


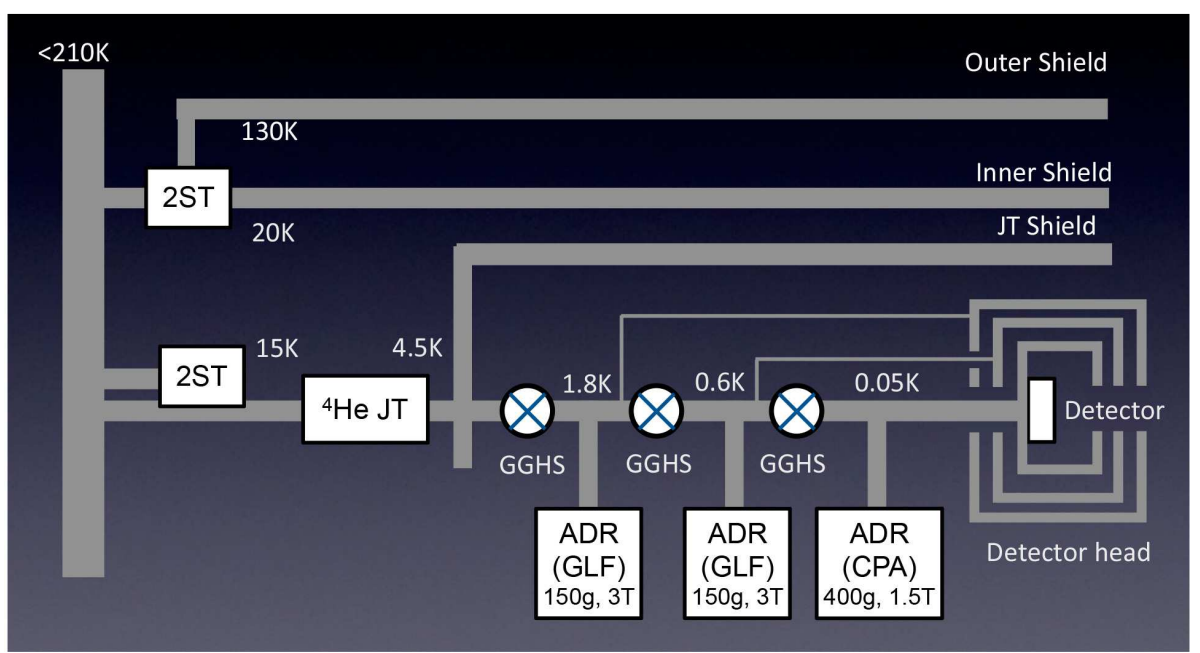

Figure 9. Cooling chain of DIOS, consisting of 2-stage Stirling coolers, ${ }^{4} \mathrm{He}$ JT cooler and 3 ADRs.

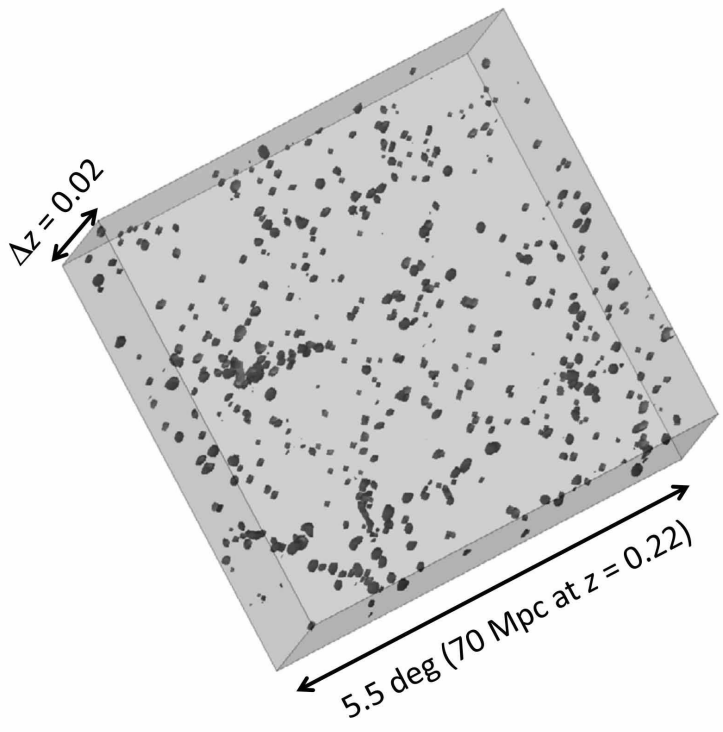

Figure 10. Expected map of WHIM based on simultaneous detection of OVII and OVIII lines above $5 \sigma$ significance for a $5^{\circ} \times 5^{\circ}$ sky, also sliced in the redshift space.

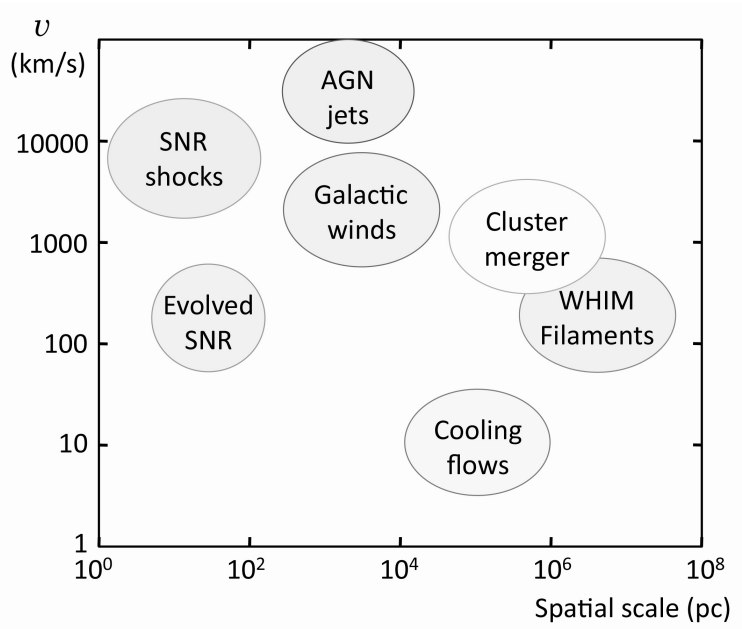

Figure 11. Large-scale gas motion expected in various systems, which will be the subject of high-resolution spectroscopy from DIOS.

and their distribution indicates the presence of large-scale filaments in several regions. ${ }^{8}$ Multi-pointing observations, such as $5 \times 5$ degree sky surveyed in 2 years, will allow us to stack a number of these images from different redshifts, thus yielding 3-dimensional distribution of WHIM. Fig. 10 shows an expected map of WHIM for a sky region of $5.5^{\circ} \times 5.5^{\circ}$ for a redshift interval $\Delta z=0.02$, and filamentary structures can be recognized.

Because of the wide field of view, combined with excellent energy resolution and low intrinsic background, DIOS can perform unique observations of extended X-ray objects which have very low surface brightness. Here, we list several examples where DIOS is expected to give new sciences.

1. Earth's magnetosphere: X-ray lines are emitted from solar-wind charge exchange process in magnetosheath and cusps in the Earth's neighborhood and DIOS will give us the structure of these emission regions. Clear separation of forbidden lines will be the key feature. 
2. Galactic interstellar medium: hot interstellar gas is expected to exhibit a Galactic fountain consisting of outgoing and infalling gases, and their dynamical motion can be studied with DIOS.

3. Supernova remnants: Distribution and motion of all the metals in SNRs will be mapped and velocity structure around shocks will be observed.

4. Starburst galaxies: Velocity and temperature of metals-rich gas outflowing into the halo region of bright galaxies will be examined in a spatially resolved manner.

5. Radio lobes: Jets are decelerated and thermalized in radio lobes, and conversion process of non-thermal energy to thermal gas will be directly studied.

6. Clusters of galaxies: Merger shocks associated with radio relics, and unvirialized gas, possibly infalling, in outer regions of clusters will be observed, and thermalization processes will be closely examined.

Fig. 11 shows targets for which study of gas dynamics in large-scale plasmas will be important. Note that most of these objects are much brighter than WHIM and their observations will take relatively shorter times. We will organize the observational program of DIOS to yield the most unique and important results in an efficient way.

\section{POSSIBLE IMPROVEMENT}

In Autumn 2013, JAXA has released a roadmap of Japanese program for space science and exploration, in which 3 mission classes are defined. The 1st category is a large mission led by ISAS, such as ASTRO-H, within a cost of 300 Myen, the 2nd is a class of Epsilon rocket launch with a cost of around 100 Myen, and the 3rd one is a mission of opportunity type with about 10 Myen per year. This allows us to make DIOS, in the 2nd category, somewhat larger than what has been considered previously. We started looking into the following possibilities.

1. Larger X-ray telescope: we may be able to extend the focal length from $70 \mathrm{~cm}$ to $1.2 \mathrm{~m}$, which makes the instrument very similar to the one proposed for the EDGE project. ${ }^{24}$ This will give the effective area close to $1000 \mathrm{~cm}^{2}$, keeping the field of view $0.7^{\circ} \times 0.7^{\circ}$. This option will significantly enhance the sensitivity to both point and diffuse sources.

2. Fast re-pointing capability: larger X-ray telescope will make observation of X-ray afterglow of gamma-ray bursts highly productive. Absorption lines from host galaxies at $z \gtrsim 3$ would be important in exploring the epoch of galaxy formation. Small gamma-ray burst monitor and intelligent attitude control system will be considered as additional features.

The introduction of the gamma-ray burst science, as well as the high-resolution spectroscopy of diffuse objects, will make DIOS a very powerful mission after ASTRO-H. It will also act as a pathfinder to Athena regarding technology development of cryogen free cooling system and TES array application including its read-out technique.

\section{STATUS AND PROSPECTS}

In 2012, Japanese high-energy astrophysics association carried out a review of 6 proposed missions, and evaluated DIOS to be "S" rank along with PolariS (polarization measurement mission). DIOS was then recommended to the astronomy and astrophysics sub-division committee of the Science Council of Japan in 2013. DIOS is also listed in the Master Plan of Large Research Projects 2014 issued by the Science Council of Japan in March 2014, as an only one future X-ray program. This makes DIOS recognized in the wide science community in Japan.

The DIOS team has joined previous mission proposals aiming for a search of dark baryons with microcalorimeter measurement of emission lines. The proposed missions are EDGE (Cosmic Vision in 2007), Xenia (Decadal Survey in 2009) and ORIGIN (Cosmic Vision in 2010), and the proposing team consists of European, US and Japanese scientists. DIOS will, thus, have international support from active groups developing TES calorimeters and X-ray optics. International collaboration will be an essential factor to realize the mission in view of raising the technology readiness, enhancing the science and sharing the emission cost.

We plan to propose DIOS to the 4th Epsilon mission with a call expected to take place in 2015-2016 and a launch in 2019-2020, if successfully selected. This would give us a nice extension of the high-resolution spectroscopy science started with ASTRO-H (launch is expected in late 2015), and technology demonstration for Athena which will be put into orbit in 2028. 


\section{REFERENCES}

[1] Shull, J. M., Smith, B. D., and Danforth, C. W., "The Baryon Census in a Multiphase Intergalactic Medium: 30\% of the Baryons May Still be Missing," ApJ 759, 23 (2012).

[2] Nicastro, F., Elvis, M., Krongold, Y., Mathur, S., Gupta, A., Danforth, C., Barcons, X., Borgani, S., Branchini, E., Cen, R., Davé, R., Kaastra, J., Paerels, F., Piro, L., Shull, J. M., Takei, Y., and Zappacosta, L., "Chandra View of the Warm-hot Intergalactic Medium toward 1ES 1553+113: Absorption-line Detections and Identifications. I.," ApJ 769, 90 (2013).

[3] Ren, B., Fang, T., and Buote, D. A., "X-Ray Absorption by the Warm-hot Intergalactic Medium in the Hercules Supercluster," ApJ 782, L6 (2014).

[4] Ohashi, T., Ishida, M., Sasaki, S., Ishisaki, Y., Mitsuda, K., Yamasaki, N. Y., Fujimoto, R., Takei, Y., Tawara, Y., Furuzawa, A., Suto, Y., Yoshikawa, Y., Kawayara, H., Kawai, N., Tsuru, T. G., Matsushita, K., and Kitayama, T., "DIOS: the diffuse intergalactic oxygen surveyor," Society of Photo-Optical Instrumentation Engineers (SPIE) Conference Series 6266, pp. 62660G (2006).

[5] Tawara, Y., Sakurai, I., Furuzawa, A., Ohashi, T., Ishisaki, Y., Ezoe, Y., Hoshino, A., Akamatsu, H., Ishikawa, K., Mitsuda, K., Yamasaki, N. Y., Takei, Y., Shinozaki, K., Masui, K., Yoshino, T., Hagihara, T., Kimura, S., and Yoshitake, H., "Status of the DIOS mission," Society of Photo-Optical Instrumentation Engineers (SPIE) Conference Series 7011, article id. 70111H, 10 pp. (2008).

[6] Ohashi, T., Ishisaki, Y., Ezoe, Y., Sasaki, S., Kawahara, H., Mitsuda, K., Yamasaki, N. Y., Takei, Y., Ishida, M., Tawara, Y., Sakurai, I., Furuzawa, A., Suto, Y., Yoshikawa, K., Kawai, N., Fujimoto, R., Tsuru, T. G., Matsushita, K., and Kitayama, T., "DIOS: the diffuse intergalactic oxygen surveyor: status and prospects," Society of Photo-Optical Instrumentation Engineers (SPIE) Conference Series 7732, article id. 77321S, 9 pp. (2010).

[7] Ohashi, T., Ishisaki, Y., Ezoe, Y., Tawara, Y., Mitsuda, K., Yamasaki, N. Y., and Takei, Y., "Status of the Diffuse Intergalactic Oxygen Surveyor (DIOS)," Society of Photo-Optical Instrumentation Engineers (SPIE) Conference Series 8443, article id. 844319, 10 pp. (2012).

[8] Takei, Y., Ursino, E., Branchini, E., Ohashi, T., Kawahara, H., Mitsuda, K., Piro, L., Corsi, A., Amati, L., den Herder, J. W., Galeazzi, M., Kaastra, J., Moscardini, L., Nicastro, F., Paerels, F., Roncarelli, M., and Viel, M., "Studying the Warm-hot Intergalactic Medium in Emission," ApJ 734, 91 (2011).

[9] Takahashi, T., Mitsuda, K., Kelley, R., Aarts, H., Aharonian, F., Akamatsu, H., and et al., "The ASTRO-H X-ray Observatory," Society of Photo-Optical Instrumentation Engineers (SPIE) Conference Series 8443 (2012).

[10] den Herder, J. W., Piro, L., Ohashi, T., Amati, L., Atteia, J., Barthelmy, S., and et al., "EDGE: explorer of diffuse emission and gamma-ray burst explosions," Society of Photo-Optical Instrumentation Engineers (SPIE) Conference Series 6688, article id. 668805 (2007).

[11] Burrows, D. N., Hartmann, D., Kouvelioutou, C., Piro, L., den Herder, J.-W., and Ohashi, T., "Xenia: cosmochemical evolution of the Universe," Society of Photo-Optical Instrumentation Engineers (SPIE) Conference Series 7732 (2010).

[12] den Herder, J.-W., Piro, L., Ohashi, T., Kouveliotou, C., Hartmann, D. H., Kaastra, J. S., and et al., "ORIGIN: metal creation and evolution from the cosmic dawn," Experimental Astronomy, 30 (2011).

[13] den Herder, J.-W., Piro, L., Ohashi, T., Kouveliotou, C., Hartmann, D. H., Kaastra, J. S., and et al., "ORIGIN: metal creation and evolution from the cosmic dawn," Experimental Astronomy 34, 519-549 (2012).

[14] Tawara, Y., Ogasaka, Y., Tamura, K., and Furuzawa, A., "Development of four-stage x-ray telescope for wide-field fine spectroscopic mission," SPIE 5168, 386 (2004).

[15] Tawara, Y., Furuzawa, A., Ogasaka, Y., Shibata, R., and Tamura, K., "The demonstration model of four-stage X-ray telescope for DIOS," SPIE 6266, pp. 62661E (2006).

[16] Tawara, Y., Kurebayashi, Y., Sugita, S., Sakurai, I., Masuda, T., Torii, T., and Matsushita, K., "The development of DIOS FXT (Four-stage X-ray Telescope)," Society of Photo-Optical Instrumentation Engineers (SPIE) Conference Series 7732, article id. 77324A, 6 pp. (2010).

[17] Yamada, S., Ezoe, Y., Ishisaki, Y., Ohashi, T., Iijima, N., Mitsuda, K., Nagayoshi, K., Akamatsu, H., Morooka, T., and Tanaka, K., "Development of Multilayer Readout Wiring TES Calorimeter for Future X-ray Missions," Journal of Low Temperature Physics 176, 310-315 (2014). 
[18] Oishi, S., Ishisaki, Y., Ezoe, Y., Abe, Y., Enokijima, Y., Hosoya, R., Ohashi, T., Mitsuda, K., Morooka, T., and Tanaka, K., "Development of Superconducting Multilayer Wiring for Large Arrays of TES X-Ray Microcalorimeters," Journal of Low Temperature Physics 167, 220-225 (2012).

[19] Ishisaki, Y., Enokijima, Y., Ezoe, Y., Ohashi, T., Akamatsu, H., Yamamoto, R., Takei, Y., Mitsuda, K., Yamasaki, N. Y., and Yamada, S., "Radiation Tolerance Evaluation of the Ti/Au Bilayer TES Microcalorimeter," Journal of Low Temperature Physics 176, 344-349 (2014).

[20] Takei, Y., Yamasaki, N. Y., Hirakoso, W., Kimura, S., and Mitsuda, K., "SQUID multiplexing using baseband feedback for space application of transition-edge sensor microcalorimeters," Superconductor Science Technology 22(11), 114008 (2009).

[21] Yamamoto, R., Sakai, K., Takei, Y., Yamasaki, N. Y., and Mitsuda, K., "Performance of Frequency Division Multiplexing Readout System for AC-Biased Transition-Edge Sensor X-ray Microcalorimeters," Journal of Low Temperature Physics (2014).

[22] Sakai, K., Takei, Y., Yamamoto, R., Yamasaki, N. Y., Mitsuda, K., Hidaka, M., Nagasawa, S., Kohjiro, S., and Miyazaki, T., "Baseband Feedback Frequency-Division Multiplexing with Low-Power dc-SQUIDs and Digital Electronics for TES X-Ray Microcalorimeters," Journal of Low Temperature Physics (2014).

[23] ASTRO-H SXS Team, Sato, Y., Shinozaki, K., Sugita, H., Mitsuda, K., Yamasaki, N. Y., Takei, Y., Nakagawa, T., Fujimoto, R., Murakami, M., Tsunematsu, S., Otsuka, K., Yoshida, S., Kanao, K., and Narasaki, K., "Development of mechanical cryocoolers for the cooling system of the Soft X-ray Spectrometer onboard Astro-H," Cryogenics 52, 158-164 (2012).

[24] Piro, L., den Herder, J. W., Ohashi, T., Amati, L., Atteia, J. L., Barthelmy, S., and et al., "EDGE: Explorer of diffuse emission and gamma-ray burst explosions," Experimental Astronomy 23, 67-89 (2009). 